\title{
Lessons Learned from Robotic Vacuum Cleaners Entering in the Home Ecosystem
}

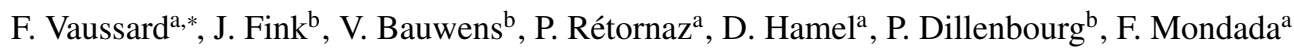 \\ ${ }^{a}$ Ecole Polytechnique Fédérale de Lausanne (EPFL), Robotic Systems Laboratory (LSRO), Station 9, 1015 Lausanne, Switzerland \\ ${ }^{b}$ Ecole Polytechnique Fédérale de Lausanne (EPFL), Pedagogical Research and Support (CRAFT), Station 20, 1015 Lausanne, Switzerland
}

\begin{abstract}
This article considers the suitability of current robots designed to assist humans in accomplishing their daily domestic tasks. With several million units sold worldwide, robotic vacuum cleaners are currently the figurehead in this field. As such, we will use them to investigate the following key question: Could a robot possibly replace the hand-operated vacuum cleaner? One must consider not just how well a robot accomplishes its task, but also how well it integrates inside the user's space and perception. We took a holistic approach to addressing these topics by combining two studies in order to build a common ground. In the first of these studies, we analyzed a sample of seven robots to identify the influence of key technologies, like the navigation system, on performance. In the second study, we conducted an ethnographic study within nine households to identify users' needs. This innovative approach enables us to recommend a number of concrete improvements aimed at fulfilling users' needs by leveraging current technologies to reach new possibilities.
\end{abstract}

Keywords:

Domestic robotics, Cleaning robots, Energy efficiency, Human factors in robotics, Human-robot interaction

\section{Introduction}

Since the early 1950s, futuristic scenarios of our daily lives at home have included robots: robot maids, robot companions, robot nannies, robot guards [2]. This vision has not substantially changed and it was only a couple of years ago that Bill Gates predicted in Scientific American that there will soon be "a robot in every home" [3]. Where are we now, however? So far, the only success of domestic robots can be noted in the field of floor-cleaning robots; millions of these devices are used to vacuum people's homes today. We do not know much yet about the long-term acceptance of domestic robots, but first exploratory studies carried out with robotic vacuum cleaners in the United States [4-6] suggest that these devices have several shortcomings that may restrict a broad user acceptance beyond initial adoption. Also, there are strong novelty effects with innovative technologies such as robots [7,8].

Today's robotic technologies are mainly driven by the technical challenges arising when a mobile robot has to perform a specific task in a loosely defined environment. However, some other topics have long been neglected in the design of robots for specific purposes, including the energy-use implications of some technical choices or harmonious integration of the robot into the user's ecosystem [9]. With technological progress, robotic vacuum cleaners (along with other domestic appliances) are now becoming widely available. The time has come to take

\footnotetext{
${ }^{25}$ A first version of this work was originally published as conference proceedings [1].

${ }^{*}$ Corresponding author. Tel.: +41 2169378 39; fax: +412169378 07.

Email address: florian.vaussard@epfl .ch (F. Vaussard)

URL: http://mobots.epfl.ch (F. Vaussard)
}

these topics into account when considering the design of future robots, as it appears essential to integrate the user into the design loop to advance these products further.

Our approach to these issues tries to be holistic and seeks synergies between current technical research and design that is acceptable for the user. For this, we integrate results from a first technical study on several robotic vacuum cleaners with findings from a second study conducted in people's homes. In doing so, we aim to advance personal robotics from both technical and user-oriented points of view. This collaborative approach brings together research from various fields.

In robotics today (in spite of a cross-disciplinary approach), the main body of the current research addresses either technical issues (perception, locomotion, or learning algorithms, just to name a few) or social phenomena independently of each other. The effectiveness of the research being performed across disciplines is muted by this process. The technical and user points of view are seldom presented side by side. Our two-sided study fills this gap.

Robotics is, by its nature, multi-disciplinary. With our proposed approach, we aim to extend the borders of the robotic community by showing how synergies can create meaningful cross-disciplinary dialogue. Ultimately, the common goal is to develop human-oriented domestic robots that enable meaningful human-robot interaction (HRI) and have the potential to improve people's quality of life.

The remainder of the paper is organized as follows: in Sec. 2 , we will state the main questions guiding both the technical and user studies. Sec. 3 will list related work to determine the state of the art in both fields, while Sec. 4 summarizes our dual methodology. The results build the core of Sec. 5, and will be 
presented using a unified outline, raising the knowledge gained up to a higher level. The conclusion of Sec. 6 will present the analysis of current robotic vacuum cleaners in light of both studies' findings, and will summarize current shortcomings. In this section, we also suggest research directions for leveraging current technologies to enhance user acceptance with targeted improvements.

\section{Motivation}

Robotic vacuum cleaners have attained a fair degree of success in the domestic robot market. The iRobot Corporation (one of the main players in this market) claims to have sold 6 million units of its "Roomba" robot between 2002 (its first release) and 2010 [10]. According to the statistics of the International Federation of Robotics [11], about 2.5 million personal and service robots were sold in 2011, an increase of $15 \%$ in numbers $(19 \%$ in value) compared to 2010 . The forecast for the period 20122015 exceeds 10 million units. This trend clearly emphasizes the growing impact of domestic robots in our homes, which creates new interaction paradigms. In parallel, the energy demand for the operation of millions of new cleaning robots will follow the same tendency.

Moreover, with the evolution of technologies, domestic robots shifted from the simple "random walk" approach towards more evolved navigation schemes, involving a localization technology at an affordable price. Up to now, no scientific study has analyzed the potential impact of these newer robots in terms of user's acceptance or energy consumption.

We have carried out two distinct but complementary studies in the present work. The remainder of this section summarizes the questions at the center of both studies, and the contributions gained by linking them together.

\subsection{Designing Efficient Domestic Robots}

The primary part of this study analyzes the current state of the art and level of achievement in domestic robotics, with a focus on robotic vacuum cleaner and energy-related topics. The robot must have several capabilities in order to fulfill its task: 1) A navigation strategy inside the environment, 2) a cleaning device, and 3) some kind of interaction with the user, at least to start and stop the cleaning process. An energy storage and management unit powers these functions. This view is illustrated in Fig. 1a. As the energy is located at the center of this robotic system, we will refer to it as an energetic agent in the course of this work.

Some research results and design choices for the various functions will impact the energy consumption of the mobile system, and thus affect its autonomy. Within this study, we aim to highlight the influence of these choices on the energy consumption, in order to design energy-wise agents that are compatible with a sustainable growth of the number of robots. As we will see, localization and navigation strategies are the main energy savers, and also bring some other benefits, but more could be achieved by adding better planning and learning. Minimization of energy consumption for robotic vacuum cleaners (and other robots) is an important topic for consideration, especially with the growth of mass-market demands and society's dependence on energy.

This paper presents an analysis of the performance of several existing robots, assessing the impact of the embedded technologies on the system's fulfillments. We present the results from a three-month study performed on a sample of seven robots, bringing together the main trends on the market. The focus is on technical metrics and therefore concerns mostly short-term issues, answering the question: "Does it work well in a domestic environment?" The key findings of this study aim at improving future designs, by identifying key technologies that enable robots to be more efficient in their environments while at the same time increasing acceptance by the end user.

\subsection{Robots in Homes Are More Than a Technical Artifact}

When deploying robots in people's homes, it is important to also consider broad human factors, as well as aspects dealing with user needs, acceptance, and social implications. Once it begins to be used by lay people in their private spaces, a robot no longer remains simply a technical artifact; rather it becomes a "social agent" [5]. A cleaning robot can have strong impacts on its direct environment ("the home"), the tasks that are related to it (e.g., cleaning), and the people in contact with it ("social actors") [4]. Fig. 1b summarizes the relations between this social agent and its surrounding environment. Ideally, these aspects should be integrated in the development of the robot using techniques such as "interaction design" or "design research" [12].

In spite of the fact that several million robotic vacuum cleaners have already been sold, not much is known about how people use and experience the presence of a service robot in their homes. Questions arise regarding the extent to which the available robotic vacuum cleaners meet user needs and expectations and how people actually use these devices. Our user study addresses these questions. The motivation of the user study was to understand users' perceptions, needs, and personal use of a robotic vacuum cleaner. We aim to identify the challenges brought on by the real world and people's unique ways of using a robotic vacuum cleaner, and to devise how design could improve these points.

With this user-centered approach, we aim to advance domestic robotic vacuum cleaners with respect to several aspects: usability, perceived usefulness, and design. These are important factors for the adoption of technology in homes [13, 14]. By understanding people's expectations and their ways of using a robotic vacuum cleaner, we can better meet users' true needs and take them into account in the process of developing these types of devices and future technologies.

To address these aspects in a holistic fashion, we conducted a six-month ethnographic study with nine households that were given an iRobot Roomba robotic vacuum cleaner. This social study was carried out in parallel to the technical study. In contrast to the technical study, the user study was motivated by the desire to shed light on the long-term implications that robotic devices might have in people's homes. In this paper, we focus on presenting results on usage and user needs of the robotic 


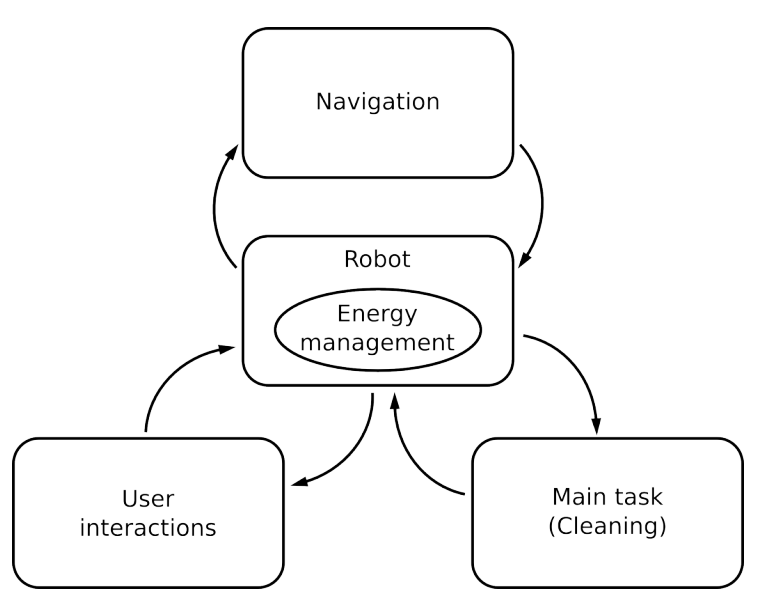

(a) Energetic agent

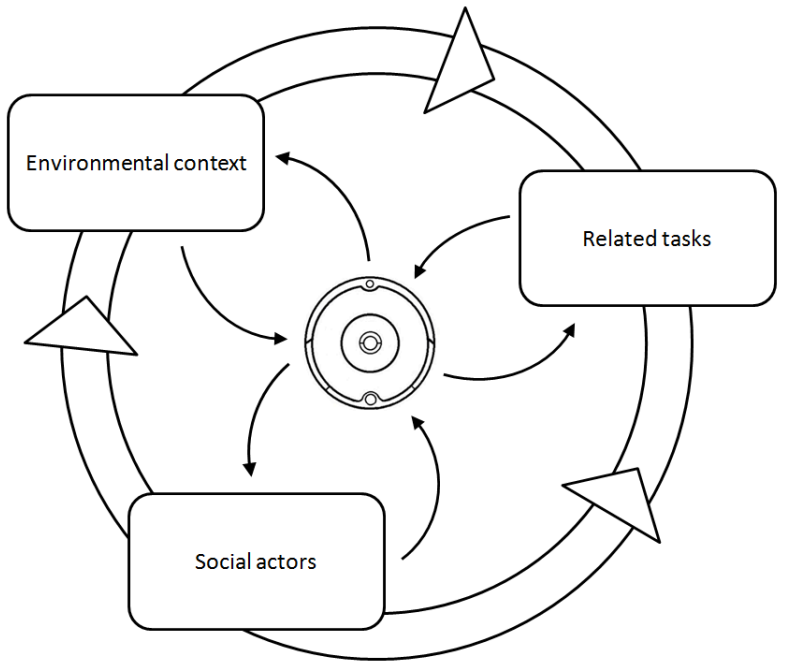

(b) Social agent

Figure 1: Our dual view of the domestic robotic agent. The agent in (a) spends its energy for an number of functions, in order to fulfill its task, and these functions in turn influence the energy consumption. The agent in (b) interacts with several elements that compose its environment.

cleaner, to leverage technical insights and provide relevant design guidelines. The detailed design and results of the ethnographic study, including long-term implications and impact of the robot on its ecosystem, are presented in another publication $[15]$.

\section{Related Work}

In the current state of the art for domestic robotics, no other study to date has attempted to provide such a close match between the scientific challenges and user acceptance of the technology. As our approach is somewhat unique, we present the related work for each topic separately.

\subsection{Technical Study}

Currently in the domestic environment, only a few types of mobile robot have been mass-produced. The first successful product, and now the most widespread, is the robotic vacuum cleaner. The first research related to creation of a robotic vacuum cleaner dates back to the 1980s [16], while the first prototype for domestic use can be dated back to 1991 [17]. Up to now, studies have compared mobile domestic robots only from an historical or purely technical point of view [18, 19]. They do not take into account the most recent trends, like the use of low-cost mapping technologies.

Other commercial applications of robotics to date have included lawn-mowing, telepresence, and pool and gutter cleaning [20]. In the literature, other examples like assistive [21] or rehabilitation robotics [22] can also be found. Most of the research has focused on key aspects such as the navigation in dynamic environments [23], or more broadly, the simultaneous localization and mapping problem (SLAM) [24-26]. Some researchers have studied performance metrics, such as the coverage of several domestic mobile robots performing a random walk [27]. Again, this does not reflect the capabilities of the latest technologies currently available. The question of energy efficiency for these kinds of appliances was only considered recently, and only to point out the lack of regulations and standards compared to other home appliances [28].

Our study proposes to fill in the current gap in the state of the art by studying a sample of the latest domestic robots, with a special focus on the energy efficiency of the overall system.

\subsection{User Study}

From a scientific viewpoint, surprisingly few evaluations of domestic service robots in real households have been carried out. For this study, we report on a pair of surveys, a set of interviews, and field studies that were carried out in people's homes. These different information-gathering techniques allowed us to develop a strong user-centered view of the currently available technology.

A pair of studies explored people's general expectations of robots and attitudes toward domestic robots [29, 30]. One important conclusion that could be drawn is that domestic robots need to be evaluated separately from robots in general, as people tend to hold different concepts of the two [6]. On one hand, people overall have rather high expectations of robots and their image of "the great mass of robotics" seems to be shaped by what science fiction and novels present to them [29, 31]. On the other hand, when people imagine a particular domestic service robot, they have no clear idea of what it could do in their 
household [29] and, accordingly, expectations of a robotic vacuum cleaner such as a Roomba are quite low [6]. Dautenhahn et al. [32] described that people want to view home robots not as friends but as machines, assistants, and servants that perform various tasks for them. Furthermore, in terms of people's perception of robots, cultural background, gender, age, and other personal factors seem to play a crucial role [29, 30].

Concerning the use of robotic floor cleaners in homes, Sung et al. described the demographic profile of Roomba owners, based on a survey among more than 350 Roomba users [33]. Against common expectations, they found that Roomba users are equally likely to be men or women and tend to be younger ( $<30$ years) with higher levels of education or technical backgrounds. Households with pets and children expressed greater satisfaction with the robot, which implies that they might use the robot differently than do those who live alone. This effect of household composition on how a robotic vacuum cleaner is used has also been noted by Forlizzi et al. [5]. Besides increasing their cleaning frequency with the Roomba, majority of Roomba owners still performs extra cleaning with their manual vacuum cleaners and most households had to make modifications to the physical layouts of their homes in order to make the robot work well $[4-6,9,15]$. These findings point out that domestic robotic vacuum cleaners could be improved to better fit in people's homes and thereby be more accepted.

Some concrete design suggestions come from Kim et al. [9] and Sung et al. [34-36]. Kim et al. deployed four different vacuuming robots in Korean homes and identified a path-planning behavior of the robot that met the assessed user needs [9]. These researchers discovered a discrepancy between the cleaning path people used when manually vacuuming and the paths chosen by the robotic vacuum cleaners. Specifically, the actual user cleans with methods unique to specific areas of the house, rather than by following a technically optimal cleaning path. Based on this, the authors suggest a robot's path planning method should use a layered map but also a cleaning area designation method reflecting each area's characteristics. This goes along with people's wish to have an intelligent domestic robot that is able to learn and adapt [6]. Furthermore, a domestic robot needs to provide a certain amount of human control, be compatible with the user's domestic environment, and take gender into consideration [34, 35]. Sung gives concrete suggestions for interaction design with everyday robots in an unpublished document [36]. On the operation level, she suggests path planning, the robot being able to learn, and reducing motor noise; on the communication level, she proposes allowing both autonomous and manual control. On the engagement level, Sung's guidelines include customization and ensuring secure service.

Our user-centered study differs from previous work in several aspects. Whereas Kim et al. [9] focused on the path-planning of humans and robots when vacuuming, we were interested in more generally exploring the usage and acceptance of vacuum cleaning robots. Kim et al. let housewives use vacuum cleaning robots for 10 days, whereas we followed up households over 6 months to be able to understand long-term effects, like user acceptance factors. In this, our study is similar to Sung et al.'s long-term study with Roombas in 30 households in the U.S.
[4]. Although it has methodological similarities [37], our work differs in a sense that it was carried out several years later (and technology had advanced meanwhile) and it was conducted in a different cultural region. However, due to our small sample size of 9 households, we are not able to generalize our results and draw on cultural differences. With our study, we are able to show several similarities but also interesting differences to the findings of Sung et al. and contribute to a better understanding of long-term effects of robots in homes.

\section{Methodology}

We used an orthogonal methodology for both of our approaches. On the one hand, seven samples of robots were tested in a synthetic environment to bring out the main factors impacting the energetic agent and its performances. On the other hand, nine households took part in an ethnographic study with a robotic vacuum cleaner to find out how people use and integrate the social agent in their cleaning routine according to their needs.

\subsection{Technical Study in Laboratory}

The sample consisted of seven robot models, ranging from the low-cost derivatives of the "Roomba" robot to recent products embedding more complex sensors and algorithms. These products target the mass market with an affordable price (between $\$ 250$ and \$600). We classified them according to their navigation strategy:

- Robots 1 to 3 (Fig. $2 \mathrm{a}-2 \mathrm{c}$ ) follow a random walk using some predefined behaviors (wall following, spirals, and obstacle avoidance for example).

- Robots 4 to 6 (Fig. 2d-2f) perform Ceiling Visual SLAM (CV-SLAM), implementing an algorithm similar to the one described by [38].

- Robot 7 (Fig. 2g) is fitted with a low-cost laser range scanner performing 2D Laser SLAM [39].

Key Questions. First, we formulate a supporting equation to help in our reasoning around the energetic agent. For a specific robot, let $p_{\text {robot }}(t)$ be the instantaneous power drawn from the battery, and $T_{\text {task }}$ be the time needed to complete the considered task. The total energy consumed to achieve the task is $E_{\text {total }}=\int_{0}^{T_{\text {task }}} p_{\text {robot }}(t) d t$. To represent the set of possible configurations, let $\vec{\alpha}$ be a set of generalized design parameters, like the type of localization algorithm. These parameters will influence both $p_{\text {robot }}(t)$ and $T_{\text {task }}$. In addition, if we take into account the efficiency of the charging electronics, $\eta_{\text {charger }}$, the parametric total energy $E_{\text {total }}$ becomes

$$
E_{\text {total }}(\vec{\alpha})=\frac{1}{\eta_{\text {charger }}} \int_{0}^{T_{\text {task }}(\vec{\alpha})} p_{\text {robot }}(t, \vec{\alpha}) d t .
$$

One goal of this study is to help design more energy-aware devices; that is, we explore the design space $\vec{\alpha}$ in order to minimize $E_{\text {total }}(\vec{\alpha})$. In Eq. 1, two functions can be minimized 


\section{Random Navigation}

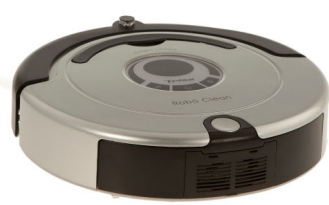

(a) Robot 1

(Trisa Robo Clean)

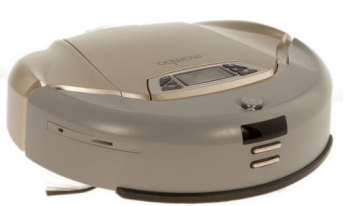

(b) Robot 2

(Primotecq Mambo)

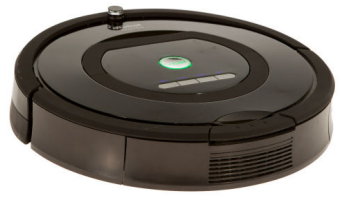

(c) Robot 3

(iRobot Roomba 770)
Ceiling Visual SLAM

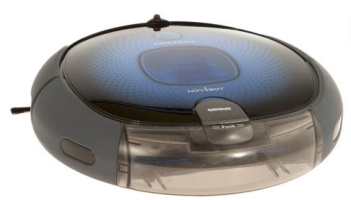

(d) Robot 4

(Samsung Navibot SR8855)

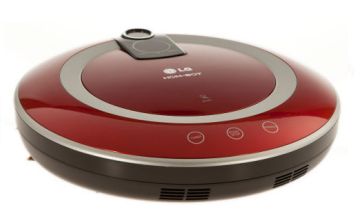

(e) Robot 5

(LG Hom-Bot VR5902)

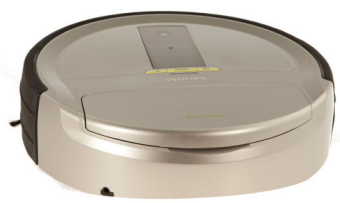

(f) Robot 6

(Philips HomeRun FC9910)

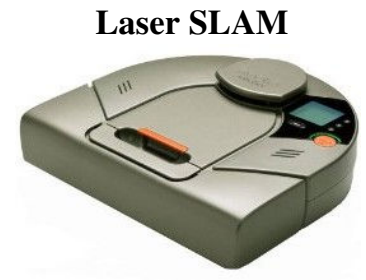

(g) Robot 7

(Neato XV-11)

Figure 2: Robots used for the technical study, sorted by their localization technology. Copyright for (a) to (f): RTS / ABE; for (g): Neato Robotics.

by varying the design parameters: $p_{\text {robot }}$ and $T_{\text {task. }}$. As we will show, neither is independent, which makes the analysis of the problem non-orthogonal.

The instantaneous power $p_{\text {robot }}(t)$ comes from the "useful" power on the one hand, and from the losses on the other hand. The required power is minimized by removing useless functions or fusing together several functions, leading, for instance, to a decrease in the number of motors used. Losses are minimized by increasing the robot's efficiency, for example by reducing the numerous electrical and magnetic losses inside the motors, as well as by reducing the Joule and switching losses inside the electronics. For a mobile robot, the energy lost when braking also accounts for a part of the total losses, and it can be partially recovered by the addition of appropriate electronics [40]. The overall control, such as obstacle avoidance, is equally important, in order for the robot to follow a smooth trajectory and avoid unnecessary braking. A modified trajectory will, in most cases, influence the completion time.

The other function to be minimized is the completion time $T_{\text {task }}$. In this case, increasing the robot's speed is often useless because it will increase the instantaneous power accordingly. Better planning and navigation are the keys for this strategy to succeed. When complete coverage is desired or required, as in cleaning, patrolling, or lawn-mowing tasks, a path planning coupled with a localization strategy will cut down the coverage time compared to a random walk approach. Recent developments in the semiconductor industry for mobile applications, coupled with algorithmic and mechatronic advances such as the laser scanner of [39], have made the simultaneous localization and mapping (SLAM) affordable for the mass market. This benefit comes at the price of extra sensors and computational power needed to achieve an efficient localization, which conflicts with the reduction of the instantaneous power.

The concern is thus to choose the best technology in order to reach a global trade-off. This article will assess, among other factors, the effect of the navigation strategy on the total energy based on measures performed with real mobile domestic robots.

Experimental Setups. During this study, we explored several performance metrics for each of the fields emphasized in Fig. 1a, making a link with the energy consumption when possible.

The navigation (Sec. 5.2) is tested in the setup depicted in Fig. 3a. It reproduces a two-room apartment with a total internal surface area of $15.5 \mathrm{~m}^{2}$ made of a concrete floor. Robots are started from their base station located in the upper right corner. The evolution of the coverage as a function of time is measured using the overhead camera (Fig. 3b) and a custom tracking software. The experiment is stopped when the robot returns to its base station or when it runs out of battery power. Between five and eleven trials were conducted with each robot.

A simple setup (Fig. 3c) is used to measure the cleaning capabilities (Sec. 5.3). It consists of a square surface ( 2 by 2 meters). Several surfacings were used: 1) smooth concrete; 2) short-pile carpet, and 3) a crack with a cross-section measuring $14 \mathrm{~mm}$ by $6 \mathrm{~mm}$. Dust was simulated using a mixture exhibiting a broad granularity range: $5 \mathrm{~g}$ wheat flour, $5 \mathrm{~g}$ of fine sand, and $5 \mathrm{~g}$ wood shavings. This was randomly spread by hand over the central square $\left(1 \mathrm{~m}^{2}\right)$ to avoid interference with the edges. The experiment was stopped when the robot returned to its base station or when the elapsed time reached 7 minutes 30 seconds. The collected material was weighed using a laboratory scale with a resolution of $10^{-3} \mathrm{~g}$. Three trials were done for each combination.

Concerning the figures for the energy consumption used throughout this work, both the global and the intrinsic instantaneous powers were measured in the setup shown in Fig. 3a. For the instantaneous power, a wireless datalogger working at $1 \mathrm{kHz}$ was hooked to the battery and used to measure the robot's in situ power $p_{\text {robot }}(t)$ during operation. As for the 


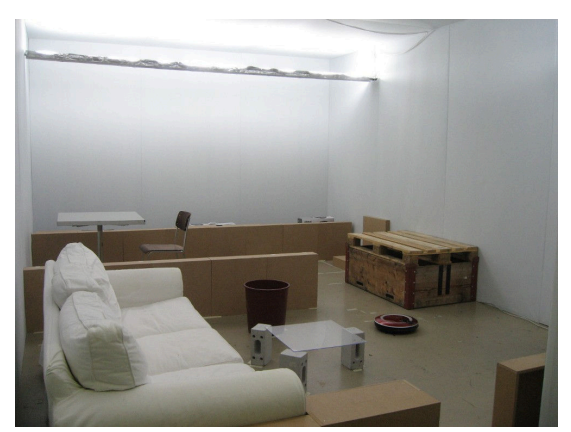

(a)

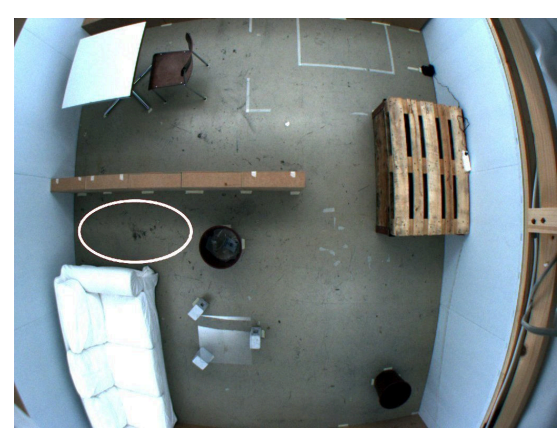

(b)

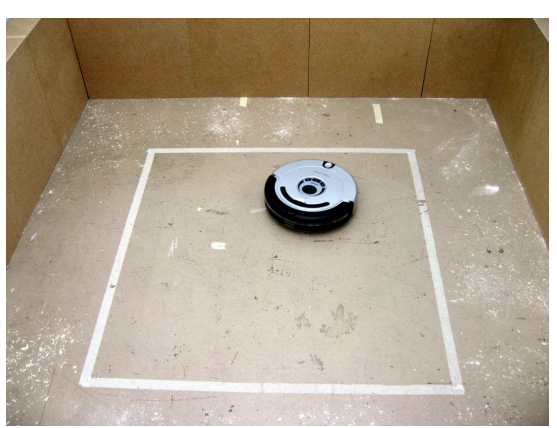

(c)

Figure 3: The experimental setups for the technical study. (a) Arena reproducing an apartment, side view. (b) Arena reproducing an apartment, from the overhead camera. The white ellipse marks an area of difficult access. (c) Simple arena for cleaning tests.

global power, a power analysis bench ${ }^{1}$ was used to measure the overall energy drawn by the charging station during a complete recharge of the battery. Dividing the overall energy drawn by the run time gives the average power consumption.

\subsection{User Study of Robot Usage "In the Wild"}

In parallel with the technical study, we deployed nine iRobot Roombas for six months in different households and studied how people used and lived with these robots in their homes. The main motivation of this long-lasting ethnographic study was to understand the process of adoption, social implications and usage patterns, and to find factors that impact the long-term acceptance of these types of devices. The identified usage patterns and user needs can be closely related to the findings from the technical study.

Course of the Study. The study was conducted from March to October 2011 and consisted of five home visits over the six months at each household in which we deployed a robot, as follows: (1) one week prior to handing out the iRobot Roomba, (2) the initial introduction, (3) two weeks after deployment of the robot, (4) two months after deployment, and (5) six months after. Several qualitative and quantitative measurements were used to capture users' feedback in order to derive usage patterns and needs: at each visit, semi-structured interviews (1 1.5 hours) were conducted in which participants were asked to describe how they used the robot, their satisfaction with it, and perceived benefits and constraints. In addition, we collected field notes, photos, and videos from the on-site observations. During the ten days before each visit, each household filled out a daily diary to document cleaning activities and Roomba usage (starting with the second visit). The methodological setup was inspired by Sung et al. [37]. We were assisted by an ethnographer during the entirety of the study.

Participants. We recruited nine households from the area of Lausanne in the French-speaking part of Switzerland. The

\footnotetext{
${ }^{1}$ Alciom PowerSpy:

http://www.alciom.com/en/products/powerspy2.html
}

sample consists of two single-person households (a 40-yearold male and a 71-year-old woman with a dog), a couple in their early 60s with two cats, and six families. The families had between one and four children; these children were aged 6 months to 18 years. Some of the families had a cat or dog. Households had culturally different backgrounds. As previous studies showed that the physical layout of the home as well as the composition of the household can impact a family's use of a robotic vacuum cleaner, we recruited a range of households living in studios, apartments, or houses, with and without children or pets, with working mothers and housewives, and with and without a cleaning service. Those households without a pet received a Roomba 520 (Fig. 4a) whereas pet-owners received a 563 PET version (Fig. 4b). We did not expect to see differences in how these two different models were used and also did not look for cultural differences impacting use, as the broader picture was of more interest to us.

Data Analysis. We collected a comprehensive and diverse set of data from our 44 household visits (five were planned at each of the nine households; one of these had to be canceled). The data considered here consist of audio transcripts from the interviews, photos, videos, written field notes, and paper-pencil diaries that people kept about their cleaning routines (descriptions about who cleans what, when, where, how, and why), as shown in Fig. 4c. The analysis of these mostly qualitative data is based on "Grounded Theory" [41, 42] and the "Method to Analyze User Behavior in Home Environment" [43]. An example of how Grounded Theory can be applied to an ethnographic study on human-robot interaction can be found in [44]. The diary entries provided quantitative data that were subjected to a descriptive statistical analysis. In total, we were able to consider $n=634$ distinct cleaning activities from the diaries, of which 193 were activities that involved the robotic vacuum and 65 involved the traditional vacuum cleaner. Our findings are based on these quantitative data and are enhanced using participants' qualitative statements. 


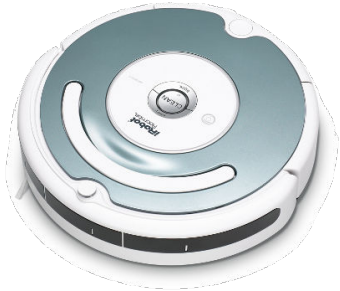

(a) Roomba 520

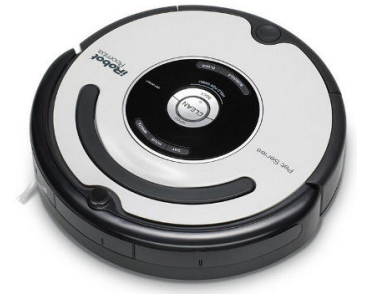

(b) Roomba 563 PET

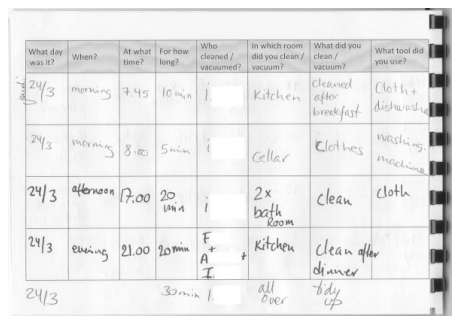

(c)

Figure 4: The experimental methodology for the user study. (a) and (b) are the robots used for the user study. Copyright: iRobot Corporation. (c) Sample of a field diary describing the cleaning routines.

\section{Results}

Results from both studies will be presented side-by-side to better highlight synergies. Sec. 5.1 will specifically focus on some of the power consumption issues. The remaining results are grouped by the research fields identified in Fig. 1a, which are the navigation (5.2), the cleaning efficiency (5.3), and the interactions with the user (5.4).

\subsection{Anatomy of the Power}

Efficient analysis of the energy issues related to a robotic system requires that one first knows the relative impact of each subsystem inside the total budget. We want to understand where the power is being used from a systemic point of view. Simple, yet informative analysis methods are used to reveal which subsystems correlate with specific power usage. The relevance of energy efficiency for the user is likewise considered.

\subsubsection{Technical Study}

An in situ analysis of the consumed power was first performed by placing an embedded, single-channel datalogger module between the battery and the robot. This module records the power consumed during the whole experiment. It gives a good, but somewhat indirect insight into the system. This method is easy to put into practice, as the battery is easily removable. Two informative plots are drawn in Fig. 5. On the other end, a multichannel datalogger would give direct figures for each subsystem, but considerable effort would be required to hack the robot's electronics.

On Fig. 5b, the startup sequence of the cleaning process for Robot 7 (Laser SLAM) is clearly visible. Starting from the idle state, the following phases can be identified: 1) the laser's spinning motor starts and stabilizes; 2) the powerful suction fan starts; 3) the main brush starts to rotate (no side brush); and 4) finally, the robot starts the driving motors and begins to clean. It can be deduced that the Laser SLAM itself consumes only $1.9 \mathrm{~W}$ ( $6.3 \%$ of the total cleaning power), compared to the cleaning subsystem, which takes $23.8 \mathrm{~W}(78.8 \%)$. The mobility accounts for $2.5 \mathrm{~W}(8.3 \%)$. For this specific robot, the power used for the navigation functions is a marginal addition compared to the cleaning device.

Such a clear breakdown, however, is not always possible with our simple method. One example of this is in the case of Robot 5 (Fig. 5a). The first small increase in power (black circle) is devised to be due to the Visual SLAM subsystem (camera and algorithm). It takes about $1.1 \mathrm{~W}$ of extra power, or $8.4 \%$ of the total power that is typically consumed by an embedded processor. All the motors start together and thus cannot be evaluated separately.

The influence of the navigation subsystem on the power budget can be further studied. Fig. 7 plots the distribution of the in situ power consumption of each robot in two working cases. Let us first consider the idle case, when the robot is turned on, but not moving. The three robots performing CV-SLAM are, not surprisingly, among the top consumers, as the additional embedded processor will need between 0.5 to $1 \mathrm{~W}$ of extra power, even when not processing any data.

When considering the cleaning case, things are completely different. The previous increase, due to the extra processing power, is largely overwhelmed by the difference due to the driving and cleaning motors. Thus, the addition of the SLAM represents only a small part of the total consumption when compared to the energy required for moving and cleaning. As we will see in Sec. 5.2, SLAM-enabled robots benefit from the acceleration of coverage, saving energy during the overall process.

A power analysis was also performed directly at the plug of the recharge station. One initial observation is the high idle power of the sole base station, with the worst result noted in the case of Robot 2 (up to $3.5 \mathrm{~W}$ ). When the charged robot is left connected to its base station, the result further deteriorates, as power consumption increases to between 3.2 and $8.1 \mathrm{~W}$ depending on the robot. Unfortunately, this type of appliance is not, at present, bound by any regulations similar to the European regulation 1275/2008 [45], which limits the standby mode to $2 \mathrm{~W}$. This represents a serious concern for these types of mass-produced electrical appliances.

The efficiency of the recharge station $\eta_{\text {charger }}$ was also computed as

$$
\eta_{\text {charger }}=\frac{1}{E_{\text {total }}} \int_{0}^{T_{\text {task }}} p_{\text {robot }}(t) d t,
$$

where $E_{\text {total }}$ is the energy consumed at the plug to recharge the robot, and $p_{\text {robot }}(t)$ is the in situ power measured on the robot during the whole process.

This efficiency figure includes the intrinsic quality of the charger as well as the storage efficiency of the battery subsystem, and varies between 0.33 and 0.84 in our study. The best two robots are the ones that use Li-ion batteries, while the oth- 


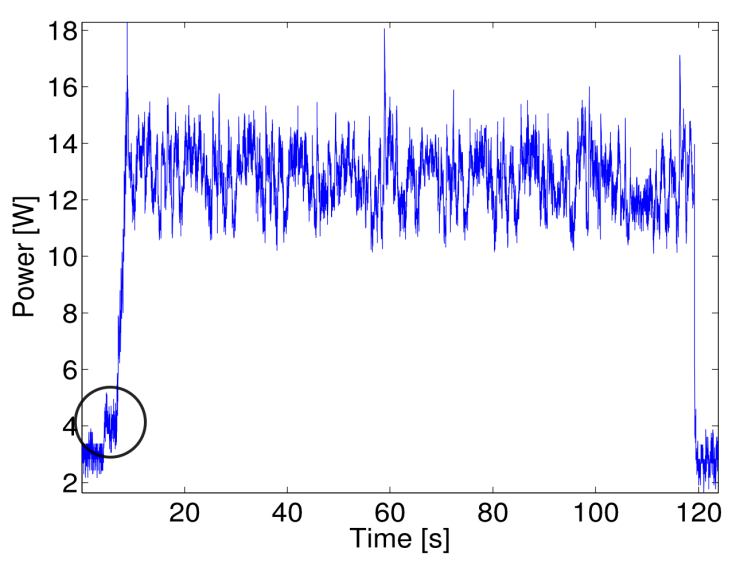

(a) Robot 5: Cleaning on concrete. The black circle pinpoints the startup of the CV-SLAM process, just before the robot starts moving and cleaning.

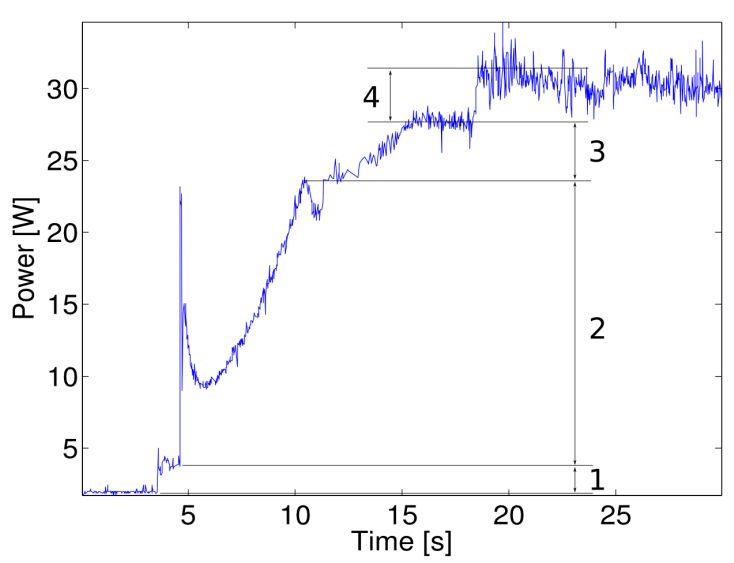

(b) Robot 7: Carpet cleaning startup processes. 1: laser 2: suction 3: brushes 4: driving motors.

Figure 5: Two plots from the in situ power measures.

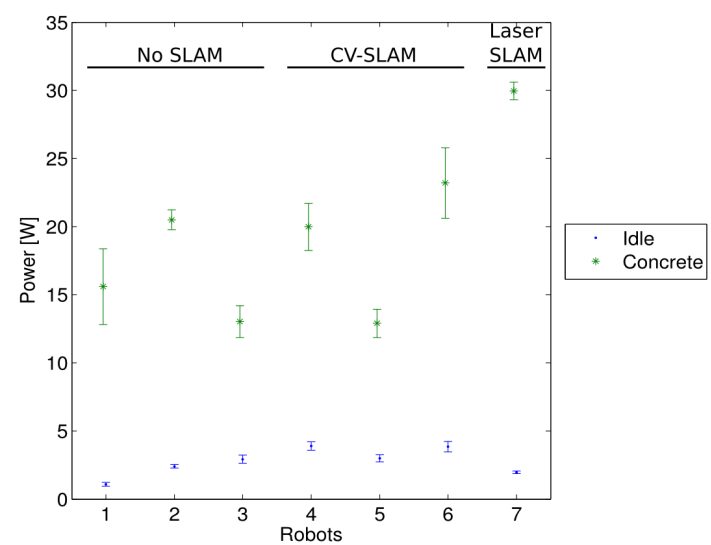

Figure 7: Task-related in situ power measured for each robot. Around 1000 samples have been used for each dataset.

ers use the Ni-MH technology. In the case of the two worst robots, more than $50 \%$ of the recharge power is already lost at the plug.

\subsubsection{User Study}

The user study confirmed the importance of the topics we addressed in our technical study. All nine households surveyed considered the energy usage of the robot, sometimes related to more practical reasons like the autonomy and the charging time. Interestingly, we did not have to bring up this topic; all of the participants independently asked for more information concerning the robot's energy use, or told us that they were concerned about the fact that the robot's charging stations needed to be plugged in at all times.

Our findings indicate that users wish to have more transparency with respect to energy usage. Some other home technologies (such as washing machines or dishwashers) already indicate their level of electricity consumption according to European standards. The reasons given by the participants for desiring reasonably low energy consumption on the part of the robot ranged from the financial importance to factors related to the environment and healthy living.

Most households completely switched off the robot and unplugged its charging station when the device was not in use to avoid overly high energy consumption. However, this hinders a programmable robot from starting a cleaning session when scheduled and thus undermines one of its most valuable facets: the ability to clean autonomously. For robotics in general, this shows how closely the aspect of reducing the energy consumption is related to user satisfaction and the acceptance of technical devices in homes.

\subsubsection{Joint Outcomes}

The user study indicated that the end-users do care about the energy consumption. This fact even leads users to overreact by unpluging the charging station to avoid idle losses. This action hinders the robot from working in an autonomous way, as a researcher would expects in the first place. On the other hand, measures performed on real hardware show poor energy performances in idle mode, with no incentives for better performance, as the current products are not bound by any regulations.

This can be fought using several tools. Better electronics, of course, is the first key to success, but the user should not be ignored. A first step goes through a restrictive regulation, but energy-awareness should also be advertised to the user using adequate feedback. The system should be able to provide information regarding past and present operations of the robot. As feedback appears to be a key issue in our study, we will discuss this point further in the coming sections.

Finally, the energy autonomy will also be of prime importance for the end-user, especially in the case of wide spaces. A robot should be able to cover the space under consideration in a single session, without requiring intervention from the user. This is directly related to the energy consumption at the heart of this work, and an improvement in the field of the energy consumption will thus directly benefit the end-users. Laser-based and vision-based mapping technologies induce an 


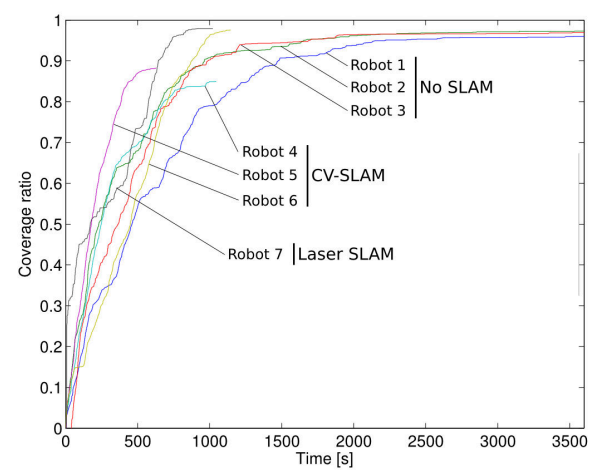

(a) Evolution of the average coverage over one hour.

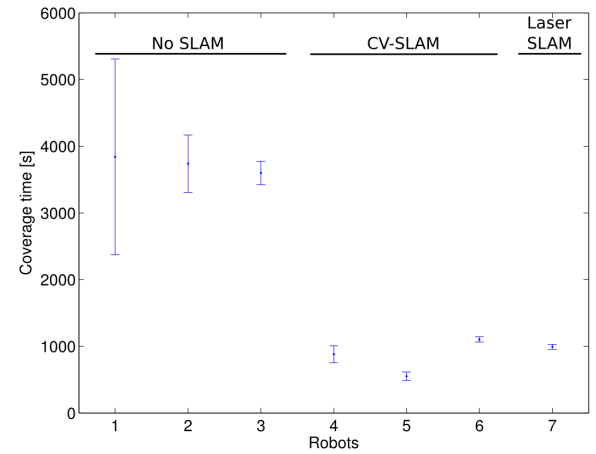

(b) Completion time for each robot.

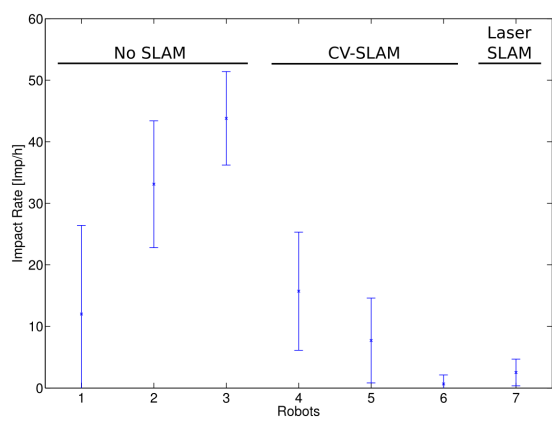

(d) Impact rate for each robot.

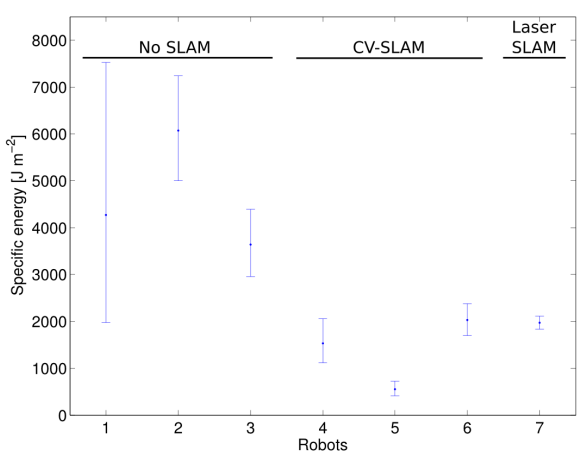

(c) The specific energy, in $\mathrm{J} \mathrm{m}^{-2}$, for each robot.

Figure 6: Analysis of navigation performance for the seven robots.

increased power consumption by 1 to $3 \mathrm{~W}$, according to our measurements. The question remains whether they will be able to save energy on the overall process.

\subsection{Navigation Efficiency}

A cleaning robot, like some other domestic robots, should not only be low-power and energy-efficient, but should also effectively cover the area of importance. Overall planning, such as the rooms to cover, the order in which the rooms should be covered, and the cleaning tasks' frequency are important topics for the user.

\subsubsection{Technical Study}

Coverage Analysis. The trajectories were recorded using an overhead video tracking system when the robot was engaged in cleaning the apartment space shown in Fig. 3a. A sample of each trajectory is shown in Fig. 8. It clearly shows distinct strategies for each robot.

To estimate the surface covered, the image analysis integrates over time the surface hidden by the robot's shape. For this purpose, the Gaussian mixture-based background segmentation of [46] is applied to the calibrated pictures. This estimation does not take into account the side brushes used by most robots, or the width of the main brush under the robot. In the results, hidden areas (like under the sofa) are not taken into account. The cleaning efficiency per surface unit is considered separately in Sec. 5.3.
The evolution of the coverage, as a function of time and averaged between all the trials, is plotted in Fig. 6a. The SLAMenabled robots seem much faster than the others. This is confirmed by the computed completion times shown in Fig. 6 b. Localization-less robots have no robust way to compute the achieved coverage. Consequently, they do not return to their base stations when the coverage reaches a steady state, and most of the time they dock to their stations after an extended period of time. On the contrary, robot 6 , which is the slowest among the robots performing SLAM, is three times faster on average than are the random walk robots.

Regarding the achieved coverage, random walk robots take time but achieve a robust coverage. On the other hand, robot 4, and to a lesser degree Robot 5 (both relying on vision), underperform compared to the others. Looking back at the image analysis, it appears that some places are harder to reach for them. One of these places is between the sofa, the intermediate wall, and the bin (white ellipse in Fig. 3b). In half of the runs, Robots 4 and 5 were unable to reach this place, thereby losing part of the coverage. On the contrary, Robot 6 was successful on all of its 11 runs, as its path planning uses thinner bands, as one can see in Fig. 8f. While some time is lost by this strategy, it gains greatly in robustness and coverage.

Specific Energy. One of our key question is the influence of design parameters on the energy consumption, and especially the navigation strategy. To answer this question, we now compare the coverage strategy, with respect to the energy. For this, we define the specific energy, which is the energy needed to cover 


\section{Random navigation}

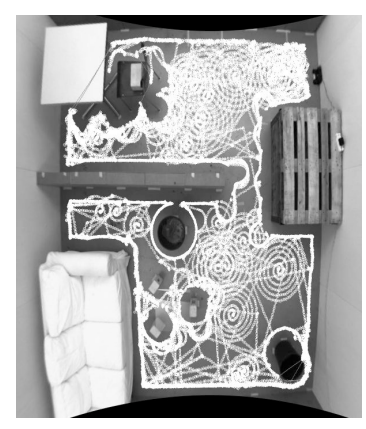

(a) Robot 1

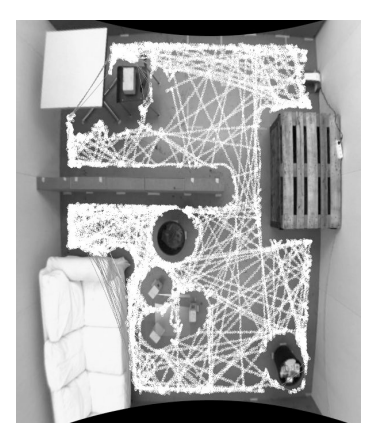

(b) Robot 2

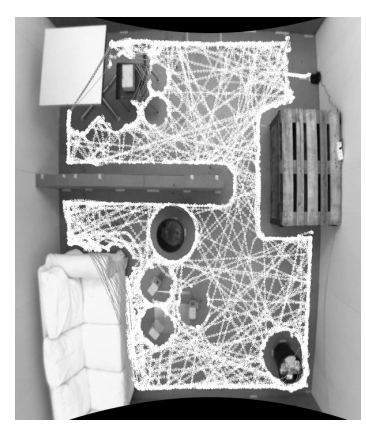

(c) Robot 3

\section{Ceiling Visual SLAM}

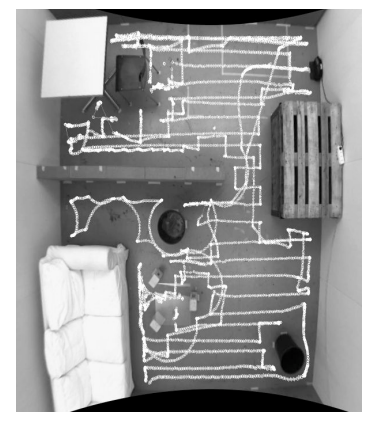

(d) Robot 4

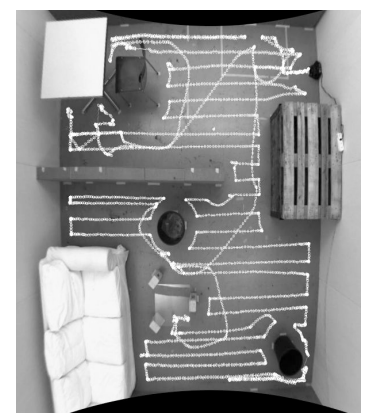

(e) Robot 5

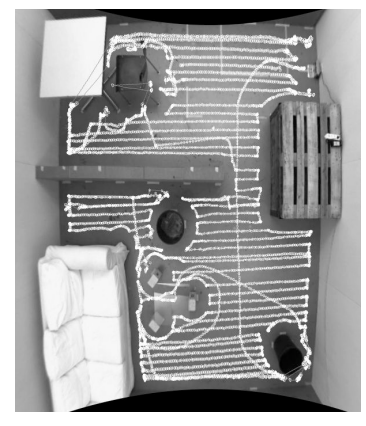

(f) Robot 6

\section{Laser SLAM}

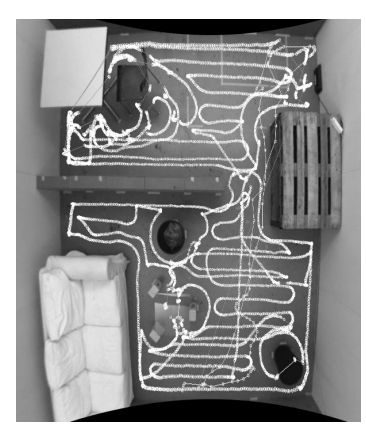

(g) Robot 7

Figure 8: Sample of the trajectories for each robot, grouped by localization strategy. 


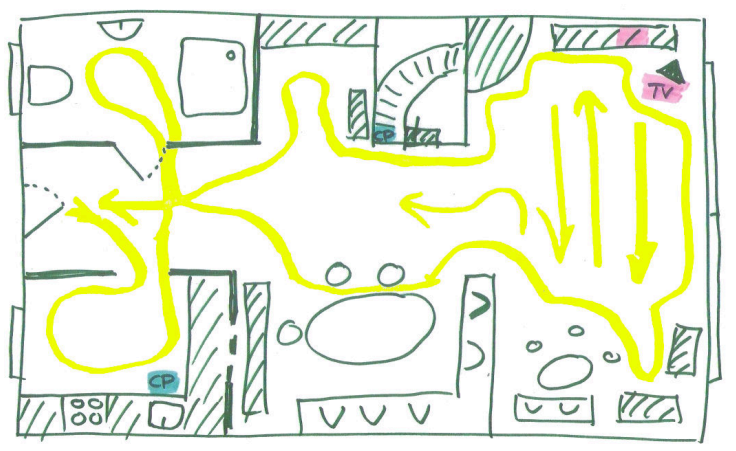

(a)

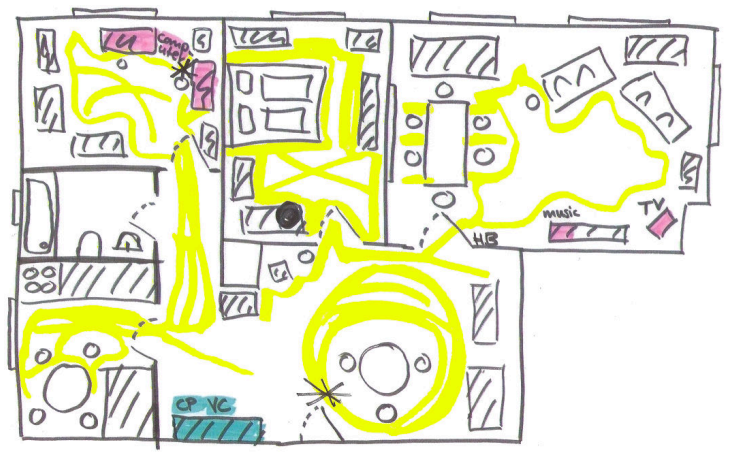

(c)

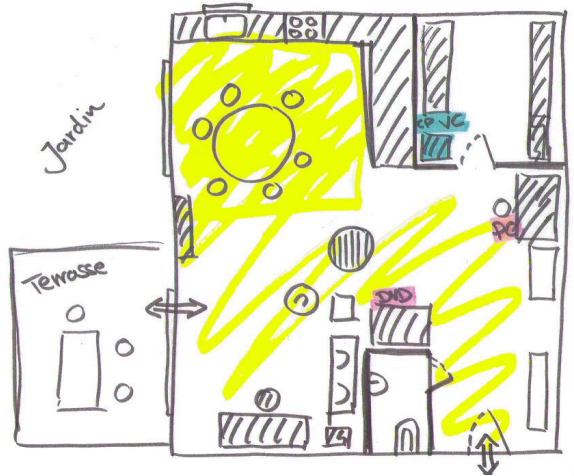

(b)

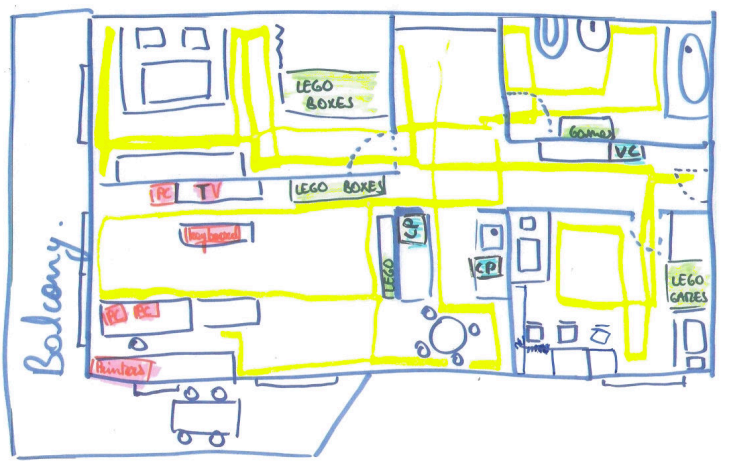

(d)

Figure 9: Sample of the expected trajectories drawn by several users.

$1 \mathrm{~m}^{2}$ of floor. It equals

$$
E_{\text {specific }}=\frac{1}{A_{\text {effective }}} \int_{0}^{T_{\text {task }}} p_{\text {robot }}(t) d t,
$$

where $A_{\text {effective }}$ is the surface effectively covered, as deduced from the previous coverage analysis. Fig. 6c shows the results, which clearly demonstrate the effectiveness of the SLAMenabled robots over those employing random-walk methods, counterbalancing the increase of power by a drastically reduced $T_{\text {task }}$. However, no clear conclusion can be drawn between CVSLAM and Laser SLAM robots, even if Robot 6 (CV-SLAM) is two times more energy efficient than are the other robots.

Impact Rate. The mitigation of the effects from the robot impacting or colliding with objects is closely related to the user's wish regarding preservation of potentially fragile objects. During the course of our experiments, an accelerometer was placed inside a ballasted dustbin to record the number of hits. This setup was located in a corner of the room (bottom right of Fig. $3 b)$. The number of impacts per hour is plotted for each robot in Fig. 6d.

Obstacle avoidance relies primarily on the proximity sensors. Most robots use infrared (IR) proximity sensors with the number of sensors employed varied between two (Robot 2) and seven (Robot 4 and Robot 6). Robot 5 was the only robot that used five ultrasound sensors (with an addition of two long-range IR sensors on the sides); Robot 7 relied on its laser scanner with a short-range IR sensor on the side to allow it to accurately follow walls.

If we take robots using only IR and ultrasound sensors (Robots 1 to 6), no sharp claim can be made regarding better performance when using a mapping technology. Robots 4 and 5 (CV-SLAM) perform comparably to Robot 1 (random walk), even if Robots 2 and 3 (random walk) perform far worse. Robot 6 (CV-SLAM), however, is the best performer in our sample. One can also notice the slower coverage in this case (Fig. 6b), as the robot clearly slows down when arriving near an obstacle, where others maintain their speed up to the point of contact. Robot 7 (Laser SLAM) also performs well, since its sensor readily provides a dense map of obstacles.

\subsubsection{User Study}

Coverage. The robot's cleaning path is closely related to the users' perception of how efficiently the robot cleans. In agreement with the results of Kim et al. [9], we found that users desire the robot to plan its path intelligently according to specific aspects such as area / sub-area of a room, floor material, and level of dirt. People generally want a cleaning robot to cover the whole floor. Participants were particularly annoyed when the robot left some spots uncleaned even in the middle of the room. For this reason, good coverage of the entire space to be cleaned is very important.

Other specifics emerged about the satisfaction (or lack thereof) of the users with the Roomba. In one instance, a mother 
expected the robot to clean the whole room in a short amount of time. She left it for only five minutes in the spacious living room, and then she switched it off to carry it to another room, only to find that the living room had not been completely cleaned. The problem, in this instance, is that the robot is being used in a way that is technically different from how it is intended to work, coupled with insufficient feedback. The robot required patience from the user side, which was cited as a difficulty for some of the households in the user study. Thus, swift coverage is also of prime importance.

Cleaning Habits. Another aspect related to the navigation of the robot is how people wish to use the robot. In agreement with Kim et al. [9], we also found that users want to use the robot according to specific spaces (for example, in the hallway, kitchen, or living room), and that users often have specific ideas concerning how a robot should progress through the cleaning of these spaces. The robot, however, does not follow such a path. Some participants also wished that the robot would clean areas in a specific order; one example of this would be to vacuum dirty spots such as under the bed at the end (also in accordance with [9]).

We analyzed participants' cleaning diaries with respect to where vacuuming was performed (Table 1). Interestingly, the robot is used in some rooms much more often than in others, and the distribution was not similar to where the manual vacuum cleaner was used. Whereas the hand-operated vacuum cleaner was most often used in the bathroom (35\%), this was the room where Roomba was used least often $(2 \%)$. Asked why, participants answered that they would be afraid of letting the robot pass over wet spots. Participants also indicated that bathrooms were tiny rooms and conveyed the belief that the robot was not adept at cleaning these spaces. Conversely, $35 \%$ of all Roomba cleanings took place in the kitchen, while it comprised only $17 \%$ of all hand-operated vacuum cleanings. An interpretation of this user behavior is that the kitchen is a place where frequent quick spot cleaning is required and that the Roomba is preferred over the manual vacuum cleaner for this kind of cleaning. A possible explanation is that it takes longer to have the manual vacuum cleaner ready for vacuuming: it needs to be taken from the closet, plugged in, adjusted, plugged out, and re-stored, whereas a robot can be used right away.

Planning Transparency. The user's viewpoint of how the robot's path planning is perceived is important for the acceptance of the robot. A robotic vacuum cleaner needs to be able to trace a smart, smooth, and efficient path that is comprehensible to some extent to humans. Before participants received a Roomba, we asked them to draw a path of how they expected the robot to move around their home (Fig. 9). Most people imagined the robot would go room by room and would spend more time cleaning in areas where more dirt was likely to be found; for example, around the dining room (crumbs from eating, etc.) (Fig. 9b and 9c). One user was convinced that the robot would go back to where she put it for the start of its cleaning path, namely at the entrance of the house (Fig. 9a). One
Table 1: Cleaning frequencies by places (in \%), when using the vacuum cleaner and the robotic cleaner, analyzed from the cleaning diaries; not all possible places are listed.

\begin{tabular}{lcc}
\hline Where? & $\begin{array}{c}\text { Vacuum } \\
\text { Cleaner } \\
(\%)\end{array}$ & $\begin{array}{c}\text { Robotic } \\
\text { Cleaner } \\
(\%)\end{array}$ \\
\hline Bathroom & 35 & 2 \\
Kitchen & 17 & 35 \\
Children room & 17 & 17 \\
Bedroom & 14 & 10 \\
Living room & 10 & 28 \\
\hline
\end{tabular}

family in which the father was a computer scientist imagined rectangular movements (Fig. 9d). Overall, people had no clear idea about how the robot would plan its path.

When people used the Roomba for the first couple of times, members of all nine households carefully watched how it moved around their homes. Most participants attempted to understand how and why the robot "decided" its path. Most households were skeptical about the Roomba's random path and one mother expressed her disappointment: "How does it decide where it goes? It's stupid, it does not see where the dirt is, it always moves away from it!" One family enjoyed the robot's unpredictability but all remaining eight households described the behavior as "stupid" and "not understandable." This unpredictability made the robot appear uncontrollable to them and six of the nine households did not want to rely on it; they were afraid to let it clean when they were not at home. The verdict was that the robot could not be trusted enough, especially in the presence of fragile objects on the ground.

This works against an early acceptance of the robot. Robotic vacuum cleaners are not entertainment robots; they are there there to fulfill a routine task. As we found in the user study, people's first impression about the robot had a strong impact on the long-term acceptance of it. Thus, preferably, a domestic service robot convinces people from the very beginning by making them understand how it works, by being transparent in its path-planning so it is "understandable," and users have fewer worries about its random path. This goes along with the aspect of "transparent robot behavior" [47]. People wish to understand the robot's navigation as this leads to the impression of having the system under control.

\subsubsection{Joint Outcomes}

The user study makes it clear that the current robots do not match users' preconceptions. Often, the user does not understand what the robot is doing, and sometimes experiences frustration or loses patience. On this point, robots using SLAM have a clear advantage by being systematic, and thus more predictable, in addition to being faster and sparing energy. From the user perspective, SLAM can play a key role to enhance user acceptance, and support the robot to provide services matching users' expectations.

Nonetheless, the feedback given by the robot could be greatly 


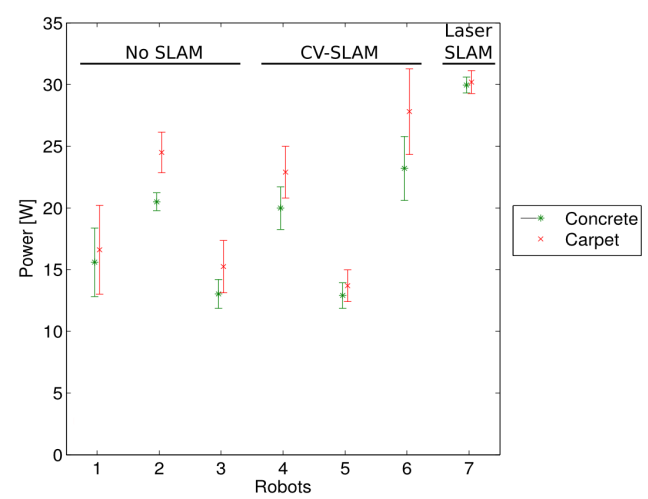

Figure 10: Analysis of the cleaning task: Surface-related in situ power measured for each robot. More than 1000 samples have been used for each dataset.

extended to improve user's awareness and to make the device more trustworthy. With the house's map at hand, the robot could communicate the estimated completion time, cleaned and uncleaned areas, or locations where it often encounters trouble and requires user's assistance. The communication medium, for example, could be an embedded screen, or perhaps even an application for a smartphone.

The user study also reveals how the cleaning habits are segmented both in time and space. Not all the areas have the same functional and emotional importance, nor do they require cleaning with the same regularity. Again, SLAM robots could reach a higher level by learning these patterns (with some feedback from the user), and fuse them with the map in order to adaptively clean. Areas with a higher density of dust can be cleaned first, and more often. Another benefit is an increased energy efficiency, as only the necessary amount of work is performed.

Finally, we have the necessary elements to answer this question: compared to a manual vacuum cleaner, does a robot perform better in term of energy? We will focus on the setup of Fig. $3 \mathrm{a}$. With a robot consuming roughly $20 \mathrm{~W}$, it will take around 15 minutes for the fastest one, equaling an expense of $18 \mathrm{~kJ}$. On the contrary, a human will take about 5 minutes with a vacuum cleaner consuming $1000 \mathrm{~W}$, equaling an expense of $300 \mathrm{~kJ}$. The robot takes about 15 times less energy. Even if people use the robot every day, they will still save energy compared to a weekly vacuuming.

\subsection{Cleaning}

Vacuum cleaning may seem to be an easy task at first, but in reality it is quite challenging, as the robot will evolve on a broad diversity of surfaces and face heterogeneity in the material to collect. The design of the embedded system must also take into account the limited power at the robot's disposal, as well as the limited space available to integrate the cleaning system. In this section, we will study the influence of the environment on the cleaning task and describe people's different cleaning strategies to ascertain how a robotic vacuum cleaner could meet them.

\subsubsection{Technical Study}

Fig. 10 shows the averaged in situ power measured for the robots when cleaning two type of surfaces, namely concrete and a short-pile carpet ${ }^{2}$. The power distribution clearly shifts upward for all the robots when cleaning the carpet. This is explained by the increased current due to the additional frictional resistance on the cleaning brushes. Cleaning a rough surface requires more power.

From the user's perspective, cleaning efficiency is one of the most important factors that determines the usefulness of a robotic vacuum. Efficiency figures were measured on three different surfaces, according to the setup described in Sec. 4.1, and averaged on three trials. These figures were then compared to the averaged in situ power previously measured. Plots are given, as a function of the surface's type, in Figs. 11a to 11c.

In the case of the concrete surface (Fig. 11a), no relationship between the cleaning efficiency and the robot's power can be seen (using a linear fit, goodness of fit $\mathrm{R}^{2}$ is only 0.018 ). Most robots scored about $90 \%$ with respect to the amount of material collected during cleaning. For comparison, the same test conducted with a manual vacuum cleaner (Dyson DC05) showed an efficiency above $98 \%$.

In the case of the carpet (Fig. 11b), cleaning efficiency $\eta_{\text {cleaning,carpet }}$ exhibits a moderate dependency on the robot's power, as shown by the linear regression

$$
\eta_{\text {cleaning,carpet }}=0.007 \cdot \bar{p}_{\text {robot }}-0.092 \quad\left(\mathrm{R}^{2}=0.56\right),
$$

where $\bar{p}_{\text {robot }}$ is the averaged in situ power. The overall efficiency remains poor (below $35 \%$ ) in all cases.

Finally, for the cleaning of the $14 \mathrm{~mm}$ by $6 \mathrm{~mm}$ crack (Fig. 11c), data are quite well explained by a linear regression

$$
\eta_{\text {cleaning,crack }}=0.041 \cdot \bar{p}_{\text {robot }}-0.47 \quad\left(\mathrm{R}^{2}=0.74\right) .
$$

In conclusion, the suction power does not really help when dealing with a flat and smooth surface. The design of the brushes is the primary concern in this case. However, suction power does become the main tool on a hard and uneven surface.

\subsubsection{User Study}

Each household cleans differently, according to the physical characteristics of their home and their personal preferences. In

\footnotetext{
${ }^{2}$ All robots were unable to clean a long-pile carpet due to the small distance between the ground and the robot's frame.
} 


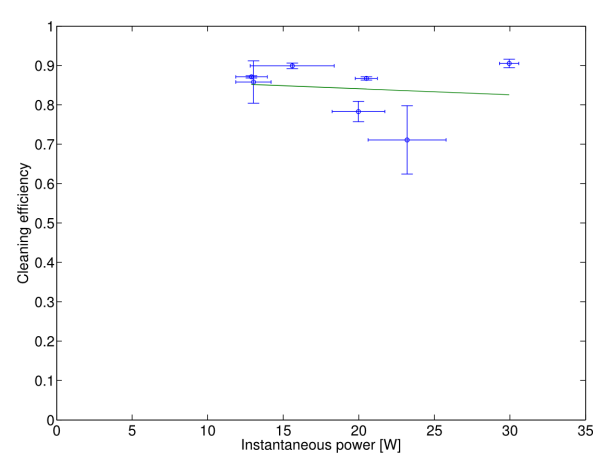

(a)

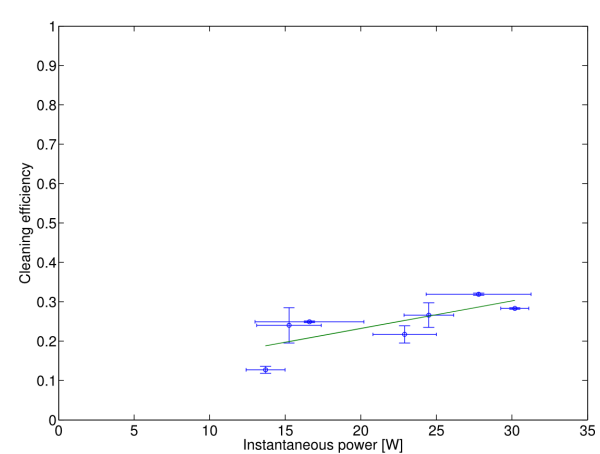

(b)

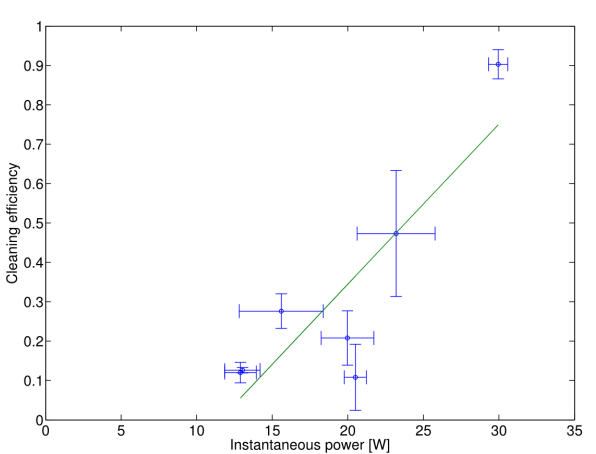

(c)

Figure 11: Analysis of the cleaning task. (a) - (c): Cleaning efficiency as a function of the robot's averaged in situ power when cleaning on concrete (a), on a carpet (b), or when cleaning a 6-mm-deep crack (c). The green line shows the linear regression performed on the data. Three trials were done each time.

cleaning, there is no "right" or "wrong." In turn, one cannot make general statements about how people clean. We would like to give a rough idea of how the households in our case study used their manual and robotic vacuum cleaners. We describe how usage patterns are determined by the housekeeper's personal conviction of cleanliness and reflected in a specific cleaning strategy.

On average, participants vacuumed their homes once per week or once every other week. Only one household with a dog that shed a lot of hair used to vacuum three times per week and one single person household only once every two months. The three households that integrated the robot in their cleaning routine used the Roomba on a daily basis, much more frequently than they used their manual vacuum cleaner. Still with the robot, the manual vacuum cleaner was used from time to time to clean well in corners. Whereas the manual vacuum cleaner was used most often on weekends (41\%), the robot was used equally often on every day of the week, with a small peak on Wednesdays (20\%). Detailed results are plotted in Fig. 12. This shows how the robot changes people's cleaning frequency, by using the robot more regularly all over the week. However, this change could only be observed in the households that adopted the robot. A household was considered as "adopter" when they kept using the robotic vacuum cleaner during the six months of the study and expressed interest in buying it if we were to take it from them.

When considering various floor surfaces, people had different opinions about how well the robot cleaned on carpet, tiles or parquet floor. However, participants consistently found the robot had difficulties with the transitions between different floor types and lost dirt it had already collected when moving onto a carpet, for instance.

Users prefer that a robotic vacuum cleaner would meet their standard of cleanliness as well as their expectations of "how cleaning is done." However, people have different attitudes towards cleaning and cleanliness. To understand better how a robot could meet a user's needs, we derived four types of cleaning strategies based on the motivation that a household shows to keep the home clean and the efforts made and time spent for cleaning [15]:

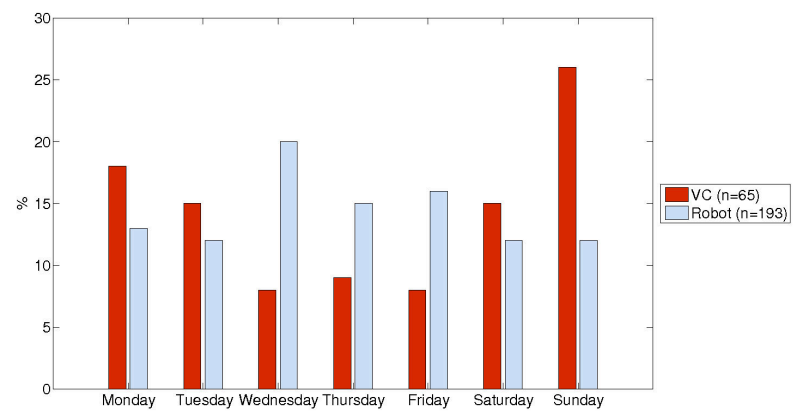

Figure 12: Frequency of use in $\%$ for the vacuum cleaner (VC) and the robotic cleaner, as a function of the days of the week. $n$ values indicate size of each dataset.

Spartan cleaners barely notice dirt and do very little about it. They have no or low motivation to clean, and hardly use the few cleaning tools they do possess. Vacuuming once every two months might be considered a typical behavior. However, in spite of this, they feel comfortable in their environments because cleaning is not of great importance to them. In our sample, one of the spartan households always set an alert on a cell phone to be reminded to vacuum; it would have been forgotten otherwise.

A robotic vacuum cleaner could more easily suit the cleaning standard of the spartan cleaners as it tends to be not very high. Given that the robot would clean efficiently, it could even replace the vacuum cleaner. Further, a cleaning robot could help spartan cleaners by automatically starting a cleaning session from time to time. A robotic vacuum cleaner for spartan cleaners could be fairly autonomous and clean while they are not at home.

Minimalistic cleaners notice dirt around the house that makes them feel a little uncomfortable (which creates some intrinsic motivation to clean). They do what is necessary but not more. Vacuuming is done only when they have time to do so. Cleanliness is not a priority.

Since minimalistic cleaners do not like to spend a lot of time tidying and cleaning, a robotic vacuum that meets 
their needs would have to be always ready for use ("opportunistic cleaning" [5]), small, and time-efficient. It also would have to work somewhat silently and discreetly to not make the user think about cleaning.

Caring cleaners really care to have a clean and nice-looking home to show guests that they have a well-working "home ecosystem" (which creates some extrinsic motivation). They like to keep the home clean (or engage a cleaning service) and enjoy cleanliness and order.

Ensuring a healthy environment for their families is central to caring cleaners. A robotic vacuum based on their needs could have a more meticulous cleaning path, with a detailed report of the collected dust (where, how much) and a visible energy-saving function.

Manic cleaners clean almost obsessively. They are very picky, notice every little piece of dirt and every blemish, and probably constantly feel the pressure to clean or tidy up. Accordingly, they permanently spend a lot of time tidying up and cleaning tasks are a priority for them. They would not engage a cleaning service because then the state of their homes would not be under their control anymore.

It is difficult to meet the standards and needs of manic cleaners as they feel a strong need to control their surroundings. For this reason, a cleaning robot would be more of an extra cleaning tool than a partial replacement for their vacuum cleaner. A less autonomous robot is suggested, which could be precisely scheduled (time and space), possibly remote controlled, and would also need to be discreet while providing perceivable value in how it cleaned (as manic cleaners would tend to observe the robot and not leave it "alone") by eventually moving slower and remaining longer in edges and corners.

This classification is based on our ethnographic study in only nine homes and is therefore eventually not generalizable. However, what is important here is to shed light on people's different cleaning strategies and also different expectations of a robotic vacuum cleaner.

\subsubsection{Joint Outcomes}

People have different expectations regarding the cleaning task. We were able to classify our households into four categories in terms of the frequency of cleanings and the level of expectations. The needs of spartan and minimalistic cleaners are already addressed by current robots. This is not the case for caring cleaners, not to mention manic ones. To address these shortcomings, the robot should be configurable, in order for the robot's design to reflect people's different needs of cleanliness and patterns of using the robot. This implies a varying level of autonomous decision (when to clean), configurable and adaptive path planning (where to clean), and the feedback given to the user (how it was cleaned).

Robots with a low power consumption clean equally well on smooth surfaces, but still have a cleaning efficiency slightly below a vacuum cleaner. On uneven surfaces, the suction power becomes important, whereas the efficiency on carpets is low in all cases. Our recommendation is to adapt the cleaning power to the type of surface using a classification algorithm.

\subsection{User Interactions}

In the following, we describe how the user and the respective robotic vacuum cleaner interact with each other. The interaction can be direct or indirect and be split into three main parts: how users operate and give commands to the robot; how the system gives feedback to the user; and other more indirect interaction with the user's and robot's shared environment, such as the noise the robot makes and its visibility during the cleaning process.

Operating the robot. The control of the robot is usually kept very simple, with one or several push buttons for immediate operation, and with a daily or weekly timer for scheduled tasks. The only operating option is to clean the whole surface, which is not the most energy-efficient, as not all the rooms would need to be cleaned with the same schedule. A rich user-friendly interface, like a touch screen, would provide the user with more control over task planning. To ease the burden on the user, a map combined with a capacitive dust sensor would enable the robot to learn where dirt was more likely to accumulate in the cleaning area. This would allow the robot a higher degree of autonomy in being able to choose the high-priority places to clean. This is related to the work of Kim et al. [9].

Feedback from the robot. The feedback is limited to a few LEDs, or a screen with very limited information. With the advent of mapping technologies, information could cover broader aspects, like where the robot is intending to go. This has the goal of reassuring the user. The robot could also provide a kind of "cleaning report," which would give more transparency to the user with respect to what the system achieved, what has to be done, and so forth. Ultimately, this could enhance acceptance and adoption of the robotic vacuum cleaner.

With respect to human-robot interaction, participants appreciated the audio-feedback (different sounds) as well as the spoken verbal feedback of the Roomba, as no other technical product they possessed would use this kind of feedback. Children, in particular, reacted very positively to the variety of sounds that the Roomba played. However, some parents were afraid this would be too engaging for the children and that their children would not stop playing with the robot. Thus, sounds could probably be more functional and less entertaining but we also found that parent's role of introducing the robot to the children influences how they approach it. Several participants suggested the robot could understand verbal commands or gestures to help it find the dirty spots in the apartment. The idea of using verbal feedback was suggested by some of the users but strongly rejected by others, as they found it intimidating. Spoken feedback can probably be integrated as an option and it is up to the user to choose it. 


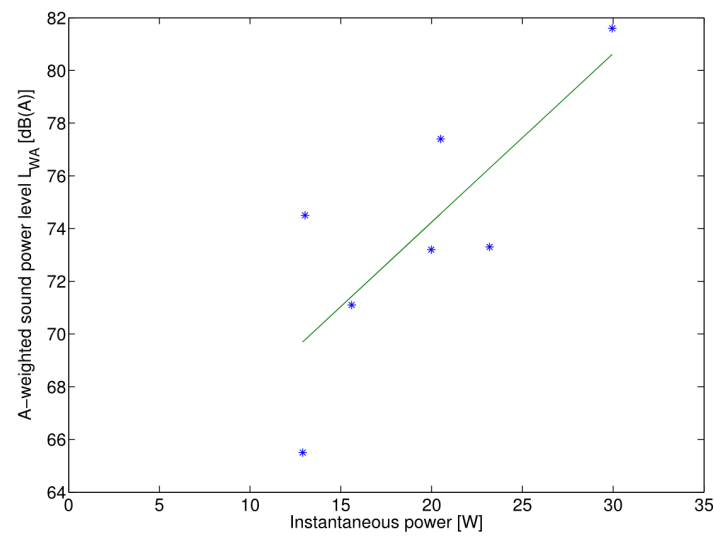

Figure 13: A-weighted sound power as a function of the averaged in situ power for each robot. The green line shows the linear regression performed on the data.

Noise. The noise emitted by the robot is also an important factor behind long-term acceptance. A noisy device will be less attractive, especially if the user wants to keep an eye on it to monitor the cleaning process. One of the families in the user study wanted to use their Roomba overnight. Although the bedrooms were located on the second floor, they still woke up due to the noise the robot made in the kitchen on the first floor. Conversely, noise is not a problem when the system works while no one is at home, which is also the intended use of a robotic cleaner.

Furthermore, people's different cleaning strategies and ways of using the robot require the system to be as quiet as possible. Some users, especially caring cleaners, would also tend to keep an eye on the robot when it was in use but would still wish to do "multi-tasking."

The total sound power level $L_{W}$ was measured according to the norm NF EN ISO 3743-1 in a reverberating room, using the mid-band frequencies between $125 \mathrm{~Hz}$ and $8 \mathrm{kHz}$. The Aweighted power level $L_{W A}$ for each robot, as a function of the averaged in situ power, is shown in Fig. 13.

The noise will depend on a broad variety of parameters, like the rotation speed of each motor, the quality of mechanics, isolation materials, brushes, and so on. But as one can see in Fig. 13 , the data can be partially modeled by the linear regression

$$
L_{W A}=0.64 \cdot \bar{p}_{\text {robot }}+61.4 \quad\left(\mathrm{R}^{2}=0.61\right) .
$$

As was shown during the crack test, the difference in power between the robots can be mainly explained by the suction power produced by the cleaning system. A low-power robot will be consequently easier to silence, making it more pleasant for the user, but reducing the cleaning efficiency of the robot on rough surfaces. This is a great opportunity for an adaptive system, which would be able to minimize the noise most of the time, while still retaining its cleaning efficiency on diverse types of surfaces.

Robot's Visibility. In contrast to a manual vacuum cleaner, a robotic vacuum cleaner is visible to the household members most of the time, even when the system is not in use. For this reason, the visible design of the robot requires special attention. Optimally, the design would fit the style of the home and likely not resemble a cleaning tool so much as some kind of home accessory. One participant explained: "I need the vacuum cleaner every single day to clean up around the table. Sure, it would be easier to have it just right next to the table. But it would not look nice. I do not want my vacuum cleaner or any other cleaning tool to be that present." The importance of a good visible design becomes increasingly important when people are hosting guests. Whereas five of the households proudly presented their cleaning robot to guests, four of the households unplugged their Roomba's charging station and put it, together with the robot, in a closet or under the bed to hide it, as they found it did not fit with the rest of the house and looked rather ugly. It is therefore necessary to put together an optimal functional shape of the robot with a good design to make the robot less intrusive, permitting it to merge with its surroundings.

\section{Conclusion}

In this paper, we presented results from an evaluation of several domestic robotic vacuum cleaners with respect to several main topics: power consumption, navigation, cleaning performance, and human-robot interaction. We did not only carry out a formal comparison of seven different robots but we also conducted a long-term user study with nine households. This holistic approach was chosen to advance further personal service robots and enhance their acceptance by merging together both sides of the robot's lifecycles: technical design and final usage.

Our methodology is based on a number of assumptions. First of all, some of the results from the user study are based on people's self-reported data. We carefully verified these data during the interviews and our on-site observations to capture "reality" as good as possible. However, these qualitative data remain subjective. Therefore, the results can only be taken as being reflective of individual cases but cannot be generalized.

Second, we only used random navigation robots for the user study, whereas the technical study also included systematic navigation robots. To investigate the impact of user's perception of the robot's path planning, another type of robot with planned navigation could have been used and complemented the results. With our study in hands, we have strong evidences that this type of robot would improve the long-term acceptance.

Finally, concerning the technical study, the environment was simulated. Although modeled after a real scenario, it still lacked some challenges encountered by robotic vacuum cleaners in people's homes. Our environment was static and constrained, while a house is generally dynamic and unconstrained. The robustness of the navigation consequently remains untested.

Nevertheless, we were able to combine the outcomes of both studies in a meaningful way. We identified concrete benefits and drawbacks of mapping technologies for domestic floor cleaning robots, on one hand regarding the amount of energy consumed by the system and on the other hand taking into account users' needs and perceptions. We found that the user's perception highly depends on how users actually use the robot, 
and that this perception in turn influences the design requirements. People wish to understand how their robotic vacuum cleaner is working ("transparency"), which is not the case with random navigation. Currently, the robot does not provide adequate feedback to the user, decreasing the chances of long-term acceptance. This could be improved by providing the user with a map of the environment, and fusing inside it information coming from the sensors like dust, energy consumption, and type of surface. The user could in turn give information to plan more precisely the tasks.

Based on people's different cleaning strategies and attitudes towards cleanliness and robots, there is no single best solution. Our evaluation shows that the manual vacuum cleaner is still more efficient for cleaning. However, this comes with a much higher energy consumption, whereas a robot is more energy efficient. When comparing different navigation strategies, the addition of a SLAM-based solution enables reduction of overall energy consumption by speeding the completion time.

Our initial question — "can a robot be a drop-in replacement to accomplish domestic tasks?" - cannot be answered out of the box. It mostly depends on how the respective product is used and thus always involves both the user and the system and their shared environment. It has to be clear that as soon as robotics tries to enter people's homes, the human will be at the center of it. Therefore, success first and foremost depends on providing solutions that match real needs.

Participants in our study wished that the robotic vacuum cleaner would solve the shortcomings of their manual vacuum cleaner, and consequently decrease the amount of work for the user. However, six of the nine households stopped using the robot after a while. Although people were at first enthusiastic and interested in trying out a robotic vacuum cleaner, the majority became disappointed as they actually assessed the robot's relevance within their own ecosystem. In this case, the rejection of robotics is not motivated by some underlying fear or negative preconceptions, but is an issue of how functional the robot is within people's ecosystem. After novelty effects had worn off, the robot became another cleaning tool with its own flaws.

We also noticed two big hurdles from the human side: a lack of trust in the robot to do its work autonomously while the homeowner is not around, and the willingness to adapt and make physical alterations to the home itself for the robot. We believe that domestic robots should be designed to minimize the need for these types of efforts.

We can suggest several practical improvements aimed at increasing the synergies between the user's needs and the robot's capabilities. It principally comes to enhancing the perceived energy efficiency, the navigation inside the user's space, and the feedback coming from the robot. These improvements leverage the available mapping technology, by pushing forward the learning and reasoning algorithms, as they are currently at a very rudimentary level.

- The surfaces to be cleaned can be classified regarding their roughness, opening doors for an adaptive suction power. Advantages to this are a reduced power consumption on flat surfaces, with a reduced noise power level. The suction power can be increased when cleaning an uneven terrain for increased efficiency.

- The dirty spots can be learned over several runs. This enables adaptive planning to be put in place, where dirty places (kitchen, living room) are cleaned with a different frequency to meet the user's needs. Energy is also spared, as only the necessary amount of cleaning is performed.

- The map enables the robot to clean by unit area, as described by [9]. This way, certain parts of the surface can be cleaned using a more refined schedule. The participants in our study expressed this aspect either as a wish for a future version of the robot, or instead as a shortcoming of the current version.

- The feedback to the user can be enhanced. On the map of the house, several layers of information could be added: what is the intended planning, what has already been cleaned, where is the dirt, are there any unreachable points, and so forth. This map can be provided using a rich interface, for example through a smartphone application. This feedback could also include estimates of time remaining until completion. This will enhance user acceptance, especially for more demanding users, by creating transparency and a feeling that the user "knows" what the robot is doing.

These improvements will be at the center of our future work, in order to provide more helpful robots for daily life. Moreover, this study allowed us to identify key parameters to reduce the energy consumption of domestic robots. By leveraging this knowledge, we are aiming at robots able to operate autonomously indoors, without having to rely on the power grid. This improvement goes towards the expectations of users and the needs of our society.

This advance implies the necessity of embedding some kind of energy harvesting into the mobile robot and/or on a charging station, providing it with energy extracted directly from the surrounding environment, as previously discussed in [48]. Light, heat, or mechanical work produced by humans could act as the primary source of energy. In any case, the available energy level will be low and highly fluctuating over time, driving the need to spare energy at the level of the complete system and to gain more information concerning the surrounding environment.

\section{Acknowledgments}

This research was supported by the Swiss National Science Foundation through the National Centre of Competence in Research Robotics. Most of the robots have been provided by François Jean-Richard from Swiss National Television (TSR). We thank Daniel Burnier (EPFL-STI-LSRO) for the datalogger used during the in situ analysis, as well as Cédric Monchâtre and Hervé Lissek (EPFL-STI-LEMA) for the acoustic measurements. 


\section{References}

[1] F. Vaussard, P. Rétornaz, D. Hamel, F. Mondada, Cutting Down the Energy Consumed by Domestic Robots: Insights from Robotic Vacuum Cleaners., in: G. Herrmann, M. Studley, M. J. Pearson, A. T. Conn, C. Melhuish, M. Witkowski, J.-H. Kim, P. Vadakkepat (Eds.), TAROS, volume 7429 of Lecture Notes in Computer Science, Springer, 2012, pp. 128-139.

[2] F. Kaplan, Everyday robotics: robots as everyday objects, in: Proceedings of the 2005 joint conference on Smart objects and ambient intelligence: innovative context-aware services: usages and technologies, sOc-EUSAI '05, ACM, New York, NY, USA, 2005, p. 59-64.

[3] B. Gates, A robot in every home, Scientific American 296 (2007) 58-65.

[4] J. Sung, R. E. Grinter, H. I. Christensen, Domestic robot ecology, International Journal of Social Robotics 2 (2010) 417-429.

[5] J. Forlizzi, How robotic products become social products: an ethnographic study of cleaning in the home, in: Proceedings of the ACM/IEEE international conference on Human-robot interaction, HRI '07, ACM, New York, NY, USA, 2007, p. 129-136.

[6] J. Forlizzi, C. DiSalvo, Service robots in the domestic environment: a study of the roomba vacuum in the home, in: Proceedings of the 1st ACM SIGCHI/SIGART conference on Human-robot interaction, HRI '06, ACM, New York, NY, USA, 2006, p. 258-265.

[7] T. Kanda, T. Hirano, D. Eaton, H. Ishiguro, Interactive robots as social partners and peer tutors for children: a field trial, Hum.-Comput. Interact. 19 (2004) 61-84.

[8] T. Kanda, H. Ishiguro, Communication Robots for Elementary Schools, Technical Report, CiteSeerX, 2005.

[9] H. Kim, H. Lee, S. Chung, C. Kim, User-centered approach to path planning of cleaning robots: analyzing user's cleaning behavior, in: Proceedings of the ACM/IEEE international conference on Human-robot interaction, HRI '07, ACM, New York, NY, USA, 2007, p. 373-380.

[10] iRobot CEO Discusses Q4 $2010 \quad$ Results, http://seekingalpha.com/article/252090-irobot-ceo-discusses-q4-2010results-earnings-call-transcript, 2010.

[11] International Federation of Robotics: World Robotics 2012 Service Robots, http://www.ifr.org/service-robots/statistics, 2012.

[12] Y. Rogers, H. Sharp, J. Preece, Interaction Design: Beyond Human Computer Interaction, John Wiley \& Sons, 2011

[13] F. D. Davis, Perceived usefulness, perceived ease of use, and user acceptance of information technology, MIS Quarterly 13 (1989) 319-340.

[14] V. Venkatesh, H. Bala, Technology acceptance model 3 and a research agenda on interventions, Decision Sciences 39 (2008) 273-315.

[15] J. Fink, V. Bauwens, F. Kaplan, P. Dillenbourg, Living with a vacuumcleaning robot. a 6-months ethnographic study., International Journal of Social Robotics (to appear).

[16] F. Yasutomi, M. Yamada, K. Tsukamoto, Cleaning robot control, in: Robotics and Automation, 1988. Proceedings., 1988 IEEE International Conference on, IEEE, pp. 1839-1841.

[17] E. Prassler, A. Ritter, C. Schaeffer, P. Fiorini, A Short History of Cleaning Robots., Auton. Robots 9 (2000) 211-226.

[18] P. Fiorini, E. Prassler, Cleaning and Household Robots: A Technology Survey., Auton. Robots 9 (2000) 227-235

[19] E. Prassler, K. Kosuge, Domestic Robotics., in: B. Siciliano, O. Khatib (Eds.), Springer Handbook of Robotics, Springer, 2008, pp. 1253-1281.

[20] I. Alonso, Service robotics, Service Robotics within the Digital Home (2011) 89-114.

[21] T. Breuer, G. R. G. Macedo, R. Hartanto, N. Hochgeschwender, D. Holz, F. Hegger, Z. Jin, C. Müller, J. Paulus, M. Reckhaus, J. A. Á. Ruiz, P.-G. Plöger, G. K. Kraetzschmar, Johnny: An Autonomous Service Robot for Domestic Environments., Journal of Intelligent and Robotic Systems 66 (2012) 245-272.

[22] I. Carrera, H. A. Moreno, R. J. Saltarén, C. Pérez, L. Puglisi, C. E. G. Cena, ROAD: domestic assistant and rehabilitation robot., Med. Biol. Engineering and Computing 49 (2011) 1201-1211.

[23] F. Yuan, L. Twardon, M. Hanheide, Dynamic path planning adopting human navigation strategies for a domestic mobile robot., in: IROS, IEEE, 2010, pp. 3275-3281.

[24] J. Leonard, Challenges for Autonomous Mobile Robots, in: Machine Vision and Image Processing Conference, 2007. IMVIP 2007. International, p. 4.
[25] S. Thrun, Simultaneous Localization and Mapping, in: M. Jefferies, W.K. Yeap (Eds.), Robotics and Cognitive Approaches to Spatial Mapping, volume 38 of Springer Tracts in Advanced Robotics, Springer Berlin Heidelberg, 2008, pp. 13-41.

[26] H. Durrant-Whyte, T. Bailey, Simultaneous localization and mapping: part I, Robotics Automation Magazine, IEEE 13 (2006) 99-110.

[27] T. Palleja, M. Tresanchez, M. Teixido, J. Palacín, Modeling floor-cleaning coverage performances of some domestic mobile robots in a reduced scenario., Robotics and Autonomous Systems 58 (2010) 37-45.

[28] A. De Almeida, J. Fong, Domestic Service Robots [TC Spotlight], Robotics \& Automation Magazine, IEEE 18 (2011) 18-20.

[29] M. Scopelliti, M. V. Giuliani, F. Fornara, Robots in a domestic setting: a psychological approach, Universal Access in the Information Society 4 (2005) 146-155.

[30] C. Ray, F. Mondada, R. Siegwart, What do people expect from robots?, in: Intelligent Robots and Systems, 2008. IROS 2008. IEEE/RSJ International Conference on, pp. 3816-3821.

[31] J. E. Young, R. Hawkins, E. Sharlin, T. Igarashi, Toward acceptable domestic robots: Applying insights from social psychology, International Journal of Social Robotics 1 (2008) 95-108.

[32] K. Dautenhahn, S. Woods, C. Kaouri, M. L. Walters, K. L. Koay, I. Werry, What is a robot companion - friend, assistant or butler?, in: 2005 IEEE/RSJ International Conference on Intelligent Robots and Systems, 2005. (IROS 2005), IEEE, 2005, pp. 1192- 1197.

[33] J.-Y. Sung, R. E. Grinter, H. I. Christensen, L. Guo, Housewives or technophiles?: understanding domestic robot owners, in: Proceedings of the 3rd ACM/IEEE international conference on Human robot interaction, HRI '08, ACM, New York, NY, USA, 2008, p. 129-136.

[34] J. Sung, H. I. Christensen, R. E. Grinter, Sketching the future: Assessing user needs for domestic robots, in: The 18th IEEE International Symposium on Robot and Human Interactive Communication, 2009. RO-MAN 2009, IEEE, 2009, pp. 153-158.

[35] J. Sung, R. E. Grinter, H. I. Christensen, "Pimp my roomba": designing for personalization, in: Proceedings of the 27th international conference on Human factors in computing systems, CHI '09, ACM, New York, NY, USA, 2009, p. 193-196.

[36] J. Sung, Design guidelines for everyday robots, http://jysung.com/robots/robot01.html, 2012. Unpublished document.

[37] J. Sung, H. I. Christensen, R. E. Grinter, Robots in the wild: understanding long-term use, in: Proceedings of the 4th ACM/IEEE international conference on Human robot interaction, HRI '09, ACM, New York, NY, USA, 2009, p. 45-52.

[38] W. Jeong, K. Lee, CV-SLAM: A new ceiling vision-based SLAM technique, in: Intelligent Robots and Systems, 2005.(IROS 2005). 2005 IEEE/RSJ International Conference on, IEEE, pp. 3195-3200.

[39] K. Konolige, J. Augenbraun, N. Donaldson, C. Fiebig, P. Shah, A lowcost laser distance sensor., in: ICRA, IEEE, 2008, pp. 3002-3008.

[40] H. Seki, K. Ishihara, S. Tadakuma, Novel Regenerative Braking Control of Electric Power-Assisted Wheelchair for Safety Downhill Road Driving, Industrial Electronics, IEEE Transactions on 56 (2009) 1393-1400.

[41] B. G. Glaser, A. L. Strauss, The Discovery of Grounded Theory: Strategies for Qualitative Research, Transaction Publishers, 1967.

[42] J. M. Corbin, A. Strauss, Grounded theory research: Procedures, canons, and evaluative criteria, Qualitative Sociology 13 (1990) 3-21.

[43] T. S. Ha, J. H. Jung, S. Y. Oh, Method to analyze user behavior in home environment, Personal and Ubiquitous Computing 10 (2005) 110-121.

[44] A. Sabelli, T. Kanda, N. Hagita, A conversational robot in an elderly care center: An ethnographic study, in: 2011 6th ACM/IEEE International Conference on Human-Robot Interaction (HRI), pp. $37-44$.

[45] Commission Regulation (EC) No 1275/2008, http://eurlex.europa.eu/LexUriServ/LexUriServ.do?uri=OJ:L:2008:339:0045:0052 $\mathrm{EN}: \mathrm{PDF}=\mathrm{rja}, 2008$

[46] P. KaewTraKulPong, R. Bowden, An improved adaptive background mixture model for real-time tracking with shadow detection, in: Proc. 2nd European Workshop on Advanced Video Based Surveillance Systems, volume 25 , pp. $1-5$.

[47] T. Kim, P. Hinds, Who should i blame? effects of autonomy and transparency on attributions in human-robot interaction, in: The 15th IEEE International Symposium on Robot and Human Interactive Communication, 2006. ROMAN 2006, IEEE, 2006, pp. 80-85.

[48] F. Vaussard, M. Bonani, P. Rétornaz, A. Martinoli, F. Mondada, Towards 
Autonomous Energy-Wise RObjects., in: R. Groß, L. Alboul, C. Melhuish, M. Witkowski, T. J. Prescott, J. Penders (Eds.), TAROS, volume 6856 of Lecture Notes in Computer Science, Springer, 2011, pp. 311322. 